\title{
EUTANASIA: CONCEPTOS DE LA FUNDACIÓN COLOMBIANA DE ÉTICA Y BIOÉTICA FUCEB, DIRIGIDOS A LA CORTE CONSTITUCIONAL (SENTENCIA T-721-17) Y AL MINISTERIO DE Salud y PROTECCIÓN SOCIAL (BORRADOR DE RESOLUCIÓN SOBRE SENTENCIA T-544-2017 DE EUTANASIA INFANTIL)
}

Nubia Leonor Posada-González* Nora Helena Riani Llano**

DOI: 10.5294/pebi.2018.22.1.11

En Colombia hay cinco sentencias de la Corte Constitucional dedicadas expresamente a la eutanasia y todas son a favor de esta alternativa destructora de seres humanos. También hubo 10 proyectos de Ley procurando la aceptación este acto violento. En esta misma sección de Persona y Bioética, en el volumen de julio-diciembre de 2015 se expuso la "Intervención en nombre de la Fundación Colombiana de Ética y Bioética FUCEB" sobre eutanasia, por el Proyecto de Ley 30 de 2015.
Contradiciendo a pocos votantes en la Corte Constitucional, el pueblo, representado en el Congreso de la República para normatizar sobre eutanasia, la ha rechazado siempre en esta instancia, lo que hace ver factible que sea necesaria una Ley estatutaria que la excluya para obedecer al reiterado mandato de la Corte Constitucional, de legislar sobre el tema, pero como el pueblo soberano ha demostrado que desea: sin destruir a seres humanos.

* PhD en Filosofía, Magíster en Educación, Espacialista en Bioética, Enfermera.

** Especialista y Magíster en Bioética, Pediatra egresada de la University of Rochester, School of Medicine. 
Estos son los dos últimos conceptos con que FUCEB intenta aportar a la reflexión bioética sobre la eutanasia, para que la toma de decisiones se base en una correlación armónica y más completa de las facultades humanas comprometidas en la gestión inteligente y responsablemente libre de sí mismo y de las relaciones con los demás miembros de la familia humana en un entorno sano y sostenible.

\section{CONCEPTO TÉCNICO SOLICITADO POR LA CORTE CONSTITUCIONAL DE COLOMBIA A LA FUNDACIÓN COLOMBIANA DE ÉTICA Y BIOÉTICA FUCEB, CITADO EN LA SENTENCIA T-721-17 SOBRE EUTANASIA}

\subsection{Caso relatado en esta Sentencia}

El 31 de julio de 2017 la Fundación Colombiana de Ética y Bioética FUCEB radicó un concepto solicitado por el Magistrado Antonio José Lizarazo Ocampo, sobre el Expediente T-6.026.987. La Sentencia fue pronunciada por la Sala Cuarta de Revisión de la Corte Constitucional, integrada por los magistrados Lizarazo Ocampo, quien la presidió, Gloria Stella Ortiz Delgado y José Fernando Reyes Cuartas.

El el 25 de octubre de 2016 se presentó la solicitud para la realización del procedimiento de eutanasia y el 18 de noviembre de 2016 se presentó la acción de tutela, de la que la Corte hace la revisión del fallo, sobre una paciente con Estado vegetativo permanente, secundario a estado pos reanimación en junio de 2008 secundario a estado epileptogénico y lobectomía en el mismo año. La paciente tenía «23 años, [...] considerada como una enfermedad "crónica, degenerativa e irreversible con mal pronóstico de recuperación" cuyas "comorbilidades pueden llevarla a desencadenar rápidamente la muerte."
[...] se elevó petición ante FAMISANAR E.P.S. para la materialización de su derecho a una muerte digna, dando aplicación al procedimiento dispuesto en la Resolución 1216 de 2015, expedida por el Ministerio de Salud y Protección Social. » (Corte Constitucional de Colombia, 2017)

El 12 de septiembre de 2017, frente al plan de análisis y manejo según el Acta de la Junta Interdisciplinaria de Cuidado Paliativo, realizada el 19 de diciembre de 2016, la EPS reporta a la Corte Constitucional: «"hasta el momento el objetivo médico ha sido prescribir medidas de soporte básico que garantizan el derecho de la paciente a una vida digna mientras se da el deceso en el transcurso natural de su enfermedad (...)" Por lo anterior, dice haberle suministrado el soporte básico, como servicio de enfermería permanente, alimentación, medicamentos crónicos y oxígeno.»

El 14 de agosto de 2017, fue hospitalizada por exacerbación de cuadro respiratorio, sumado a posible obstrucción intestinal. El 15 de septiembre de 2017 se observó "una paciente en mal estado general con apneas prolongadas, cianosis central. Se considera inminencia de muerte, se acompaña a la familia en el proceso." Se cancela el reinicio de nutrición y se justifica su hospitalización para "acompañamiento en la parte final de la vida”. (Folios 313 a 318). Murió el sábado 16 de septiembre de 2017.

\subsection{El concepto de FUCEB para la Corte Constitucional}

Conforme a lo solicitado por la Corte, especialmente interesada en el consentimiento sustituto, FUCEB respondió: 
El "consentimiento sustituto" es un subtema del consentimiento informado que compromete las principales capacidades de quien lo vaya a dar, y que debe ejercitarse buscando el mayor beneficio, en cuanto humano, para el miembro de la familia humana representado.

En el primer Considerando del Preámbulo de la Declaración Universal de los Derechos humanos se lee: "Considerando que la libertad, la justicia y la paz en el mundo tienen por base el reconocimiento de la dignidad intrínseca y de los derechos iguales e inalienables de todos los miembros de la familia humana" (Organización de las Naciones Unidas ONU, 1948).

Asumir la responsabilidad de dar con acierto un "consentimiento sustituto" reclama conocer qué es un ser humano. También es necesario dilucidar en qué consisten la "dignidad intrínseca" y los "derechos iguales". Éstos, por ser "inalienables”, no se incluyen dentro de las competencias de quien practica un consentimiento, bien sea propio o sustituto.

[...] cada individuo de la especie humana, desde que tiene por cuerpo una célula hasta que termina su ciclo vital natural, es una unidad con las dimensiones biopsico-social y espiritual y, por lo tanto, un ser que en cada instante de su existencia posee el mismo valor en cuanto humano; este valor es superior a la totalidad de la energía de la que proceden la materia inerte y los individuos vivos no humanos. Esta condición lo hace responsable del resto de la naturaleza y, en especial, de sí mismo y de los demás Miembros de su especie. Lo anterior es comprobable por las conductas humanas que superan el alcance de las leyes de la biología, quedando así demostrado que cada individuo humano tiene una condición que es superior a la sola mate- rialidad de su cuerpo, por ejemplo, estudia la acción voluntaria y, en el universo conocido, la voluntad es una facultad exclusivamente humana: voluntariamente se puede perdonar, de corazón, al injusto agresor. La inteligencia supera la capacidad de conocimiento de lo real hasta poder llegar a nociones como la de la nada; el ser humano aspira a una felicidad que abarca y trasciende las limitaciones temporales de la biología y, por lo tanto, ésta no es su fuente, sino medio de expresión de la fuente, aunque el cuerpo humano tiene el valor superior de ser, en parte, causa, cauce y ocasión para que se puedan percibir con el propio cerebro y otras partes, las manifestaciones de la inteligencia, la voluntad y el núcleo afectivo). (Fundación Colombiana de Ética y Bioética FUCEB, 2009).

La noción de la nada trasciende el universo mismo, por lo que tiene una cocausa diferente de la energía que lo constituye. El ser humano suele llegar al conocimiento de las causas a través de los efectos. De la noción de la nada se deduce que el espíritu no es un producto de la energía y, por lo tanto, es simple, no tiene partes, no se descompone, no termina y no hay motivo razonable para valorar a un ser humano en cuanto humano, según una gradualidad biológica de su crecimiento y desarrollo o con base en el deterioro biológico, sino que todos valen igual porque cada uno es una unidad corporeoespiritual. Por eso se usa la palabra "Dignidad" para reconocer el espíritu humano que por ser un bien, valor o perfección superior al bien de la biología, común a los demás seres vivos, enriquece la perfección del cuerpo humano por su realidad de ser un cuerpo personal -un ser espiritual es persona-, de modo que lo que se hace al cuerpo humano se hace a la persona humana, pues no hay cuerpo humano que no sea una persona humana. En el mundo conocido, nadie se hace mejor persona sin su cuerpo. 
Entre los referentes marco de esta definición está la Declaración Universal sobre Bioética y Derechos Humanos, que señala en sus considerandos 15 y 16 :

Teniendo presente que la diversidad cultural, fuente de intercambios, innovación y creatividad, es necesaria para la especie humana y, en este sentido, constituye un patrimonio común de la humanidad, pero destacando a la vez que no se debe invocar a expensas de los derechos humanos y las libertades fundamentales,

Teniendo presente también que la identidad de una persona comprende dimensiones biológicas, psicológicas, sociales, culturales y espirituales (Organización de las Naciones Unidas ONU, 2005)

De lo anterior se deduce que cualquier cuerpo vivo de la especie humana tiene igual valor que cualquier otro de la misma especie, y tiene un valor superior a cualquier cuerpo de otra especie. Esta universalidad del valor de cada cuerpo humano vivo, es decir, de cada ser humano o persona humana, de modo independiente a la etapa y circunstancias de su ciclo vital, hace que sea un deber de cada persona con uso de razón, cada familia y sociedad, y de cada Estado y cualquier otra institución, reconocerlo como inalienable ante los deseos propios o de terceros.

Este es un motivo por el que no existe el derecho a disponer de la propia vida ni la de terceros. En esto no existen excepciones. Es verdad que, por bienes espirituales, que son superiores, hay personas que dan su vida biológica, como los soldados que defienden la libertad y la paz de su patria, pero este no es el caso de la eutanasia, que es una opción inmanente, nunca es trascendente porque quien reconoce que también es espiritual, sabe que todo ser limitado es causado por otro y todo ser del universo conocido es limitado, luego la respuesta sobre el bien que es cada ser humano y su indisponibilidad -para los seres vivos vivir es ser-, es necesariamente externa a los seres humanos.

Una prueba de la unidad corporeoespiritual en que consiste cada ser humano es los efectos psicológicos de destruir a seres humanos inocentes e indefensos, de modo independiente a la etapa y circunstancias de su vida. Dos ejemplos de esto en el caso de la eutanasia son investigaciones científicas hechas en Bélgica y en Holanda, que demuestran que los médicos que practican la eutanasia se insensibilizan tanto respecto al respeto que merecen sus pacientes por ser humanos, que ellos mismos reconocen que llega un momento en que solo obedecen las normas legales de respeto a la autonomía de los pacientes cuando supone para los médicos algún riesgo de ser acusados. El efecto de esta insensibilidad es que, si no se valora lo mayor -con el consiguiente respeto que merece cada uno de estos seres corporeoespirituales, por el bien o perfección en que consisten-, tampoco se respeta lo menor: normas legales. El resultado es que, por cada paciente al que se le practica la eutanasia en nombre de su autonomía, hay otro al que se le practica sin valorar para nada dicha autonomía, porque los médicos se sienten con mejor criterio que los pacientes, para determinar cuándo es mejor destruir a estos enfermos.

Lo que hay de fondo en la ideología del deseo, de la que el tema de la eutanasia es uno entre varios, es la absolutización del deseo ciego, descontextualizado de la persona que desea, que acaba siendo lesionada o destruida por sí misma o por terceros. En nombre de la autonomía, se acaba con la autonomía por el camino de darle más valor al deseo propio o ajeno, que a la persona que es 
sacrificada, destruyéndola, en nombre del deseo propio o el de terceros.

Al practicarse en la intimidad, nunca los estados que han aprobado la eutanasia, han sido capaces de controlar este fenómeno: que por cada persona que la pide y se le practica, hay otra que no la solicita y también se le practica.

La calidad de vida de quien es corporeoespiritual no termina por ser simple el espíritu. Por eso la calidad de desarrollo existencial debe buscarse, en la medida de lo posible, mediante cuidados proporcionados y adecuados, pero dicha calidad supone la vida y el derecho a vivir para todos y para cada uno, sin discriminación y sin abandonos.

Se anexan dos investigaciones científicas que respaldan lo afirmado y que son parte integrante de este concepto técnico.

\section{CONCEPTO DE LA FUNDACIÓN COLOMBIANA DE ÉTICA Y BIOÉTICA FUCEB, SOBRE LAS SENTENCIAS DE LA CORTE CONSTITUCIONAL Y SUS IMPERATIVOS AL MINISTERIO DE SALUD, PARA REGULAR LA ORDEN DE LA APLICACIÓN DE LA EUTANASIA A NIÑOS Y ADOLESCENTES}

El cinco de marzo de 2018 FUCEB, representado por la Dra. Nora Helena Riani-Llano, Especialista y Máster en Bioética, Pediatra egresada de la University of Rochester, School of Medicine, intervino en el Foro organizado por el programa doctoral de Salud Pública de la Facultad de Medicina de la Universidad Nacional de Colombia en Bogotá, acerca del borrador de la Resolución del Ministerio de Salud y Protección Social, "Por medio de la cual se reglamenta el procedimiento para hacer efectivo el derecho a morir con dignidad de los niños, las niñas y los adolescentes [...] en cumplimiento de la sentencia T-544 de 2017 de la Corte Constitucional. "

El Presidente de FUCEB, Dr. Gabriel Adolfo Centanaro Meza, Neurólogo, especialista y c. PHD en Bioética, envió al Ministerio de Salud un pronunciamiento sobre la posición de la Fundación expuesta por la Dra. Riani: Al ser la eutanasia la destrucción de un ser digno en cuanto humano, es una contradicción afirmar que sean dignos las decisiones autónomas y los actos con que, directa o indirectamente, es causada. La persona no es digna por tener autonomía, sino que el valor de la autonomía es el de ser una de las perfecciones de la persona, que es responsable por su buen o su mal ejercicio. (Negrilla en el original)

La Fundación Colombiana de Ética y Bioética FUCEB, agradece a la Universidad Nacional, su diligencia por fortalecer la reflexión interdisciplinaria acerca de las decisiones de la Corte Constitucional y el Ministerio de Salud y Protección Social, sobre la práctica de la eutanasia en niños y adolescentes.

\subsection{Desarrollo}

Los mismos encargados, de las ramas jurídica y ejecutiva (supliendo curiosamente la legislativa), y que supuestamente se oponen a la desprotección del Derecho a la vida que es inviolable según la Constitución, son quienes están ordenando y exigiendo que los médicos desconozcan su Juramento y su solemne promesa de velar con sumo respeto por toda vida humana (de acuerdo con la actual Declaración de Ginebra, versión 2017, de la Asociación Médica Mundial). 
El Sr. Alejandro Gaviria Ministro de Salud y Protección Social (MSPS) tuvo cuatro meses para responder a la corte constitucional, pero al resto de colombianos nos ha dado 5 días; e inmediatamente después pretende reglamentar con urgencia (este viernes) la Eutanasia de Niños y Adolescentes (N\&A).

Solicitamos más tiempo para analizar esta delicada y preocupante propuesta del MSPS presionado por la Corte Constitucional (CC). Pedimos ampliar la deliberación de manera que más expertos pediatras y subespecialistas, psiquiatras y psicólogos clínicos pediátricos; representantes de la sociedad civil, de las familias colombianas, los bioeticistas clínicos; mas todas las otras personas y organizaciones formales pertinentes, puedan manifestarse. Ojalá también los médicos pediatras y demás especialistas afines, que entienden las graves y complejas implicaciones (más la peligrosa pendiente resbaladiza) se sumen a este compromiso de proteger a los N\&A. Quienes prestan servicios sanitarios, en cabeza de los galenos, son cuidadores antes que sanadores y definitivamente antes que meros dispensadores de "terapias".

Preguntamos ¿Qué médico realmente puede pensar que es terapéutico acabar con una vida? Provocar la muerte de otro, peor si el otro se entregó como paciente, lo consideramos una falta grave a la moral. La eutanasia implica irrespetar la confianza que se otorga al médico, a la medicina y a la especial dignidad de la persona humana.

La opción acertada que todos debemos exigir al estado colombiano en estos casos es la de los Cuidados Paliativos, más importantes aún en elcampodelaPediatría(CPP), conbuena disponibilidad y real accesibilidad. Sin una adecuada implementación efectiva y sostenida de éstos, y no la falencia admitida en el mismo borrador, consideramos que está faltando al derecho fundamental a la salud.

Es nuestro deber cumplir nuestros enaltecidos roles en la sociedad, reaccionando a esta gravísima falta. La verdadera vocación del médico es la de curar y aliviar, o cuando menos, acompañar, dando inconmensurable valor y reconocimiento a la dignidad de cada ser humano aún en su etapa final y más si se trata de menores de edad, ya que no tienen la noción ni el trayecto vivencial suficiente para tomar esta decisión de manera independiente, y más cuando están doblegados y atemorizados a causa del sufrimiento y el dolor. ¡Es una tristeza que ellos llegasen a preferir morir porque les hemos fallado!

Consideramos moralmente reprochable permitir por normativa que las clínicas y hospitales se vean en la obligación de imponer al personal sanitario, encabezado por el médico, esta funesta actividad.

\subsection{Elementos conceptuales y legales que nos preo- cupan del borrador de la resolución del MSPS}

Sin proporcionado énfasis en la necesidad de ofrecer CPP suficientes, oportunos y accesibles, es decir terapias de alivio y confort; amarra este servicio a la provocada muerte eutanásica. Tanto el MSPS y la CC como el público (que clama), saben las grandes falencias en el país para garantizar CPP. Sí hay legislación, pero en la práctica falta demasiado.

La evidencia sugiere que los que piden eutanasia son quienes no reciben cuidados y aportes realmente suficientes, tanto técnico científicos como físicos, psíquicos, emocionales y aún espirituales; es decir, son a quienes el personal sanitario, las familias y la comunidad en general les ha quedado mal. 
Precisamente por esto existe una normativa en contra del maltrato infantil que abarca negligencia y abandono (igual que para el adulto mayor), que si bien está conceptuado para los entornos sociofamiliares también es de consideración por todos los actores adultos responsables; más, si son prestadores de servicios sanitarios. No faltará quienes terminan temiendo ir a un hospital o médico pensando que se les practicará eutanasia.

Salen con frecuencia en los medios de comunicación y en las redes sociales publicaciones testimoniales de personas y familias que se arrepienten de pensar en la eutanasia o que denuncian sentir coerción o influencia indebida por parte de terceros.

Falta evidencia científica y social, cuantitativa y cualitativa, en la población de N\&A relacionando factores como el estrés, la depresión, el abandono y otras causas semejantes, con los correspondientes deseos de por sí cambiantes, de vivir o morir.

En una situación de sufrimiento o dolor que altera el estado físico, psíquico y emocional a causa de enfermedad o condición adversa, es muy difícil constatar la capacidad objetiva de cualquier persona, mucho más la de N\&A. Esperar que con base en un "deseo" manifestado bajo el estrés del momento se dispense un acto irreversible que pone final a la vida (no clasificable como terapia) es desacertado y cuando menos imprudente y arriesgado.

Es cierto, pero del pasado, que los pacientes, las familias y en general la sociedad tuvo miedo por el encarnizamiento, ensañamiento u obstinación terapéutica después que se desarrolló rápidamente la ciencia-técnica médica intensivista. Este asunto ha sido correctamente tratado, con modificaciones actitudinales por parte de los mé- dicos tratantes intensivistas, ayudados por los 48 años que lleva la disciplina bioética (ejemplos: ajustes del esfuerzo terapéutico, principio de doble efecto, orden de no reanimar por motivos ortotanásicos, propios de los Cuidados Paliativos, clínicas del dolor). Así que esto deja de ser un argumento válido a favor de tener que recurrir a la eutanasia.

Hay estudios que evidencian las infortunadas pendientes resbaladizas en Holanda donde practican la eutanasia involuntaria a recién nacidos y la eutanasia voluntaria a N \& A. Podríamos estudiar más a fondo estos fenómenos y aprender de ellos antes de seguir el mismo error.

Muchos médicos no ejercen la relación médico-paciente (RMP) deliberativa en la cual el MD es casi un amigo o un mentor que guía hacia un acuerdo de manejo compartido, mediante una prudente y sabia persuasión que pone en consideración los valores del paciente y su familia. La autonomía del paciente es respetada pero la decisión final es compartida y no cómplice de daño al paciente.

Contrario a esto sería ejercer, dentro de los vínculos convenidos con las IPS, usualmente solo con una RMP informativa técnica, sumisa o interpretativa como consejería contractual. Sería despersonalizar, dejar que finalmente toda la decisión quede a manos de la autonomía del paciente. Así, el uso de una RMP inadecuada también influye negativamente (en un proceso tan delicado y definitivo) hasta el punto de entorpecer los procesos valorativos para dictaminar la capacidad y la voluntad anticipada del menor.

La autonomía del médico, que ejerce su profesión en forma libre e independiente a través de las IPS con o sin 
subordinación laboral, se ve intimidada y opacada por las normativas impuestas. Esto altera su capacidad para abordar, analizar, profundizar y refutar ante exigencias negativas como esta (eutanasia de $\mathrm{N} \& \mathrm{~A}$ ), que a la vez lo desgastan y le aumentan los riesgos de burnout, desilusión, depresión, desespero, adicción y suicidio.

Muchos expertos en psicología clínica pediátrica, puericultores y expertos en crianza incluyendo padres de familia, no estarán de acuerdo con las definiciones de capacidad de los menores en este borrador; ni estarán de acuerdo con el empoderamiento letal de la autonomía que promueve esta norma. Falta entonces mayor revisión.

En el punto 8.4 el procedimiento dice que es prerrequisito descartar el síndrome de cuidador cansado (SCC) para aplicar la eutanasia. Nos preguntamos ¿qué cuidador no sufre el SCC ante un ser querido gravemente o crónicamente enfermo? En este orden de ideas siempre habría esta contraindicación.

Todo ciudadano colombiano tiene el derecho constitucional a ejercer lo que su conciencia le dicta, pero también tiene derecho a su intimidad y privacidad. Esto hace que pueda objetar a conciencia sin tener que manifestarse "por escrito y debidamente motivada" ante un comité como lo exige esta norma. No solo el médico puede hacerlo sino todo colombiano, lo cual puede entorpecer y afectar un servicio sanitario por negaciones a participar; y estarían con todo el derecho de hacerlo.

2.3 Elementos que nos preocupan del borrador en cuanto a las clínicas y hospitales (las IPS)

Si bien la estructura jurídica institucional no puede objetar en conciencia porque esta es siempre personal, los que constituyen las instituciones pueden ejercer -y se les debe respetar-, su autonomía a través de las misiones, visiones y valores con los que se identifican como equipo de personas en su lugar de trabajo. Ellas responden ante los entes supervisores controladores, pero estos no pueden incidir con imposiciones a la conciencia expresada en los estatutos, misión, visión y valores elegidos autónomamente como comunes y organizacionales.

Las IPS que cuentan con servicios de mediana y alta complejidad, pero no tienen habilitado un servicio de CPP simplemente no podrán cumplir las determinaciones estipuladas.

La OMS y las organizaciones expertas internacionales señalan y denuncian las falencias a nivel mundial de cuidados paliativo (CP) y el mismo borrador de norma reconoce que - independiente a que ya existe norma de CP-, a nivel nacional nos quedamos cortos. Sabemos todos sobre las barreras de acceso y oportunidad de manejo y la no buena disponibilidad de fármacos aún con expertos paliativos disponibles.

Muchas de las IPS que no cuentan con los expertos exigidos: médico, abogado, psiquiatra o psicólogo clínico con "título de posgrado de un programa que forme en N\&A" prerrequisito del mal llamado "servicio". Tampoco existen los programas formales completos o suficientes en el país ni están homologando diligentemente este tipo de estudios cuando se hacen en el exterior.

También observamos que las IPS tendrán dificultades cuando los médicos vinculados tratan de ejercer entendiblemente cierta autonomía con respecto a procedimientos antiéticos porque no obedecen a la verdadera 
terapéutica; o cuando la mayoría manifiesta objeción de conciencia.

Entonces, las IPS se encontrarán en situaciones o confrontaciones si tratan de cumplir la norma. Por cumplirla no se puede obligar a los médicos a ir en contra de la más reciente versión (2017) de la Declaración de Ginebra que aún les pide prometer solemnemente velar con sumo respeto por la vida humana.

Las IPS prestan servicios sanitarios en un país sin la suficiencia económica ni el grado de desarrollo, pero ninguno es vanguardista practicando el procedimiento eutanásico, que además es tan cuestionado moral y éticamente por la humanidad entera, más con los niños. Nos preocupa que el economista a cargo de MSPS, en vez de preguntarse por qué tantos países no han optado por esta desacertada imposición, la promueva sin oponerse al mandato mortal para niños.

Las IPS están siendo obligadas una vez más, tanto del sector privado como público, a prestar "servicios" mortales impuestos pero éticamente cuestionables.

Finalmente, y en directa relación con estos temas que atañen al SGSSS de Colombia, debemos señalar que aún no existe el Consejo Nacional de Bioética - cuya constitución está decretada y va atrasada - y por ende es muy limitado el espacio adecuado (prestándose a grandes sesgos) para dirimir estos temas con actores representantes de todos los sectores. ¿Quiénes y qué tantos fueron los expertos, naturales o jurídicos, que asesoraron al Ministro de Salud antes de elaborar el borrador? ¿Hubo buena representación pluralista e interdisciplinaria?
Tanto por la experiencia clínica, multidisciplinaria y por ser académicos (con muchas publicaciones sobre el tema general de eutanasia y sobre la humanización en salud) nuestros miembros declaran éstas y muchas más inquietudes. Siendo algunos miembros de FUCEB corresponsables de servicios de salud claves en ciudades principales y estando provistos del tiempo y de las experiencias que supuestamente forman "expertos", sus miembros pueden decir que han participado de manera asistencial y de apoyo en muchísimos casos sin que hubieran percibido que haga falta ofertar este cuestionado "servicio" que reta y posiblemente desvirtúa una vez más tanto el deber ser como el noble ejercicio de la profesión médica.

Creemos que todos coincidimos en que sí es un derecho fundamental de todo ser humano, llegar a la finalización de esta vida muriendo revestido de dignidad; $y$, seguimos comprometidos con todos los actores y con todos los colombianos a que lo logremos; más si se trata de ayudar a los más vulnerables incluyendo especialmente los N\&A. Pero, señalamos como desacertada y aún equivocada la interpretación sobre la autonomía, de consecuencias mortales, que la Corte Constitucional de Colombia y el Ministerio de Salud y Protección Social vienen promoviendo: ninguna de estas instancias está por encima del derecho fundamental de cada ser humano a vivir, a aprender a valorar y cuidar, y a que se le cuide, su vida. Las opciones terapéuticas, compasivas, técnico científicas y éticas de los servicios sanitarios en cabeza del personal médico especializado en atención infantil y adolescente, no tienen por qué incluir la eutanasia. Acompañaremos siempre a morir con dignidad, pero también a vivir lo mejor posible, aún durante el proceso natural de morir. 
Hasta aquí lo entregado al Ministerio de Salud y protección Social.

\section{CONCLUSIÓN}

El país necesita un nuevo proyecto de Ley Estatutaria que prohíba la violación del derecho que es fundamento de todos los demás, a la inviolabilidad de la vida de cada ser humano durante su ciclo vital completo, consagrado en el Artículo 11 de la Constitución Política y coherente con que, al promover su destrucción, se niega que el ser humano sea fin en sí mismo, la libertad pierde su razón de ser y el Estado de Derecho se queda sin su punto de apoyo. 